\title{
Curcumin photodynamic effect in the treatment of the induced periodontitis in rats
}

\author{
Letícia Helena Theodoro $^{1} \cdot$ Marcio Luiz Ferro-Alves $^{1} \cdot$ Mariéllen Longo $^{1}$ • \\ Marta Aparecida Alberton Nuernberg ${ }^{1}$. Renata Pironato Ferreira ${ }^{1}$ • Adriele Andreati ${ }^{1}$. \\ Edilson Ervolino $^{2} \cdot$ Cristiane Duque $^{3} \cdot$ Valdir Gouveia Garcia $^{1}$
}

Received: 13 February 2017 / Accepted: 11 June 2017 / Published online: 3 July 2017

(C) Springer-Verlag London Ltd. 2017

\begin{abstract}
This study assessed the effect of curcumin as a photosensitizer in antimicrobial photodynamic therapy (aPDT) for the treatment of induced periodontitis in rats. Periodontitis was induced via a ligature around the mandibular first molar on the left side of 96 rats. The ligature was removed 7 days later, and the animals were randomized into four groups: NT, no local treatment; CUR, irrigation with curcumin solution $(40 \mu \mathrm{M})$; LED, irradiation with a light-emitting diode (LED, InGaN, 465-485 nm, $200 \mathrm{~mW} / \mathrm{cm}^{2}, 60 \mathrm{~s}$ ); and aPDT, irrigation with curcumin solution $(40 \mu \mathrm{M})$ followed by irradiation with LED. Eight animals from each group were euthanized at 7,15 , and 30 days post-treatment. Treatments were assessed using alveolar bone loss (ABL) in the furcation region using histological, histometric, and immunohistochemical analyses. Rats treated with aPDT exhibited less ABL at 7 days compared to the NT group, moderate pattern immunolabeling for osteoprotegerin at 30 days, and a pattern of immunolabeling for RANKL from moderate to low. Treatments resulted in smaller numbers of TRAP-positive cells compared to the NT group. aPDT as monotherapy using curcumin as a photosensitizer and LED as the light source was effective in the treatment of induced periodontitis in rats.
\end{abstract}

Letícia Helena Theodoro

letheodoro@foa.unesp.br

1 São Paulo State University (Unesp), School of Dentistry, Araçatuba, Department of Surgery and Integrated Clinic, Division of Periodontics, Araçatuba, São Paulo, Brazil

2 São Paulo State University (Unesp), School of Dentistry, Araçatuba, Department of Basic Science, Araçatuba, São Paulo, Brazil

3 São Paulo State University (Unesp), School of Dentistry, Araçatuba, Department of Paediatric and Social Dentistry, Araçatuba, São Paulo, Brazil
Keywords Curcumin $\cdot$ Photochemotherapy $\cdot$ Periodontitis · Rats

\section{Introduction}

Periodontitis is an inflammatory disease, which results in the destruction of periodontal tissues of the affected teeth, and is a result of the host response to microbial biofilm containing pathogenic bacteria [1]. Scaling and root planing (SRP) is the conventional mechanical treatment of periodontal disease. However, the effectiveness of this procedure is limited in areas with difficult access, such as areas of bi- or trifurcations and deep pockets [2]. Supporting therapies were proposed as adjuvant therapies to SRP, such as antimicrobial photodynamic therapy (aPDT). aPDT is defined as a photochemical oxygen-dependent reaction that involves the absorption of energy by the photosensitizer from a visible light source. This chemical process generates reactive oxygen species, such as singlet oxygen, which damage microorganisms without promoting bacterial resistance [3]. Previous studies demonstrated promising results of aPDT in the reduction of microbial viability [4, 5] and control of experimental periodontitis via a reduction in the inflammatory process [5] and alveolar bone loss (ABL) $[4,6-10]$.

Several recent clinical studies in humans evaluated the effect of aPDT as an adjunctive therapy in the treatment of chronic periodontitis [11-18]. However, some of these studies used phenothiazine as photosensitizing agent and a low-power laser, which did not demonstrate any clinical advantages of aPDT in periodontitis treatment $[12,15,18]$. Different photosensitizers in different concentrations are described in the literature, such as malachite green, phthalocyanines, porphyrins, phenothiazines, cyanines, 
acridines, and herbal and chlorins dyes [10, 18, 19]. Phenothiazine photosensitizers, such as methylene blue and toluidine blue $\mathrm{O}$, are the most commonly used in aPDTs [11-17].

Curcumin is a compound isolated from Curcuma longa, and it has been used for centuries as a pigment in food and medications with a variety of indications, including the treatment of injuries, liver disease, and antimicrobial control [20]. Curcumin exhibits no toxic effects in vitro or in vivo, and it has a broad absorption peak in the range 300-500 nm (430-435 nm max) [21]. Curcumin exerts a potent phototoxic effect on many microorganisms [22]. Several investigations suggested that curcumin exhibited potential for therapeutic application because of its anti-inflammatory, antioxidant, antimicrobial, antifungal [23, 24], and anti-cancer [21] effects. Some studies also suggested that these effects are strengthened by combination with light [25-27]. This compound provides great economic advantage, ease of use, and high efficiency [26], which attracted researchers that are interested in exploring its use in photochemistry and photobiology [26, 28-30].

Few studies evaluated the effects of photodynamic therapy using curcumin as a photosensitizer and LED (light-emitting diode) as the light source [26-30]. Therefore, the present study evaluated the photodynamic effect of curcumin in the treatment of experimental periodontitis.

\section{Material and methods}

\section{Animals}

The present study used 96 adult male Wistar rats (Rattus norvegicus albinus) weighing approximately 200 to $300 \mathrm{~g}$. The animals were housed in plastic cages with food and water available ad libitum throughout the experimental period. The Animal Research Ethics Committee of the Araçatuba School of Dentistry, São Paulo State University (UNESP) approved all protocols, which were performed in compliance with the current standards adopted by the Brazilian College of Animal Experimentation (Process \#00725-2012).

\section{Experimental induction of periodontal disease}

Animals were anesthetized with ketamine $(70 \mathrm{mg} / \mathrm{kg}$; Vetaset - Fort Dodge Iowa, USA) and xylazine $(6 \mathrm{mg} / \mathrm{kg}$; Coopazine Coopers São Paulo, SP, Brazil) via intramuscular injection prior to all surgical procedures. Periodontitis was experimentally induced using a cotton ligature (current Cotton $\mathrm{n}^{\circ} .24$; Coats Corrente, São Paulo, SP, Brazil) placed in a submarginal position on the left mandibular first molar of each animal [7]. The ligatures were removed after 7 days, and the animals were divided into four groups of 24 animals each. The rats were randomly assigned using a computer-generated table to one of four treatment groups: NT, no local treatment; LED, irradiation with LED; CUR, irrigation with curcumin solution $(40 \mu \mathrm{M})$; or aPDT, irrigation with curcumin solution $(40 \mu \mathrm{M})$ followed by LED irradiation.

\section{LED treatment}

Irradiation with an indium gallium nitride (InGaN) LED (Kon-lux Kondortech Dental Equipment Ltda., São Carlos, $\mathrm{SP}$, Brazil) with a wavelength of $468-485 \mathrm{~nm}$ was performed at a power density of $200 \mathrm{~mW} / \mathrm{cm}^{2}$ and a total energy density of $15 \mathrm{~J} / \mathrm{cm}^{2}$. The tip of LED output $\left(0.78 \mathrm{~cm}^{2}\right)$ was positioned perpendicularly to the long axis of the tooth on the buccal side of the left mandibular first molar. Animals in the LED group received irradiation immediately after ligature removal during $60 \mathrm{~s}$. Irradiation in aPDT group was performed following $60 \mathrm{~s}$ of irrigation with the curcumin solution during $60 \mathrm{~s}$.

\section{CUR and aPDT treatment}

The natural CUR photosensitizer (Fluka Co.) was obtained from Sigma-Aldrich (St. Louis, MO, USA). A stock solution of CUR $(600 \mathrm{uM})$ was prepared and diluted in distilled water to a concentration of $40 \mu \mathrm{M}$ [27]. The curcumin solution $(1 \mathrm{ml})$ was slowly dropped into the periodontal pocket around the left mandibular first molar using an insulin syringe in the CUR group. The curcumin solution $(1 \mathrm{ml})$ in the aPDT group was slowly dropped into the periodontal pocket around the left mandibular first molar using an insulin syringe, and the LED was applied $60 \mathrm{~s}$ later (pre-irradiation time) on the buccal side of the left mandibular first molar perpendicularly and in contact with the gingival tissue during $60 \mathrm{~s}$, as described in the LED group.

\section{Experimental periods}

Eight animals from each group were euthanized at 7, 15, and 30 days after local treatment of experimental periodontitis via a lethal dose of thiopental $(150 \mathrm{mg} / \mathrm{kg}$; Cristália, Itapira, SP, Brazil). Jaws were removed and fixed in $4 \%$ formaldehyde in $0.1 \mathrm{M}$ phosphate buffer (pH 7.4) for $48 \mathrm{~h}$.

\section{Histological and immunohistochemistry processing}

The left hemimandibles were dissected, fixed in $4 \%$ formaldehyde for $48 \mathrm{~h}$, and demineralized in a solution of $10 \%$ ethylenediaminetetraacetic acid (EDTA) for 60 days (Sigma Chemical Co., St. Louis, MO, USA). Semi-serial sections $(4 \mathrm{~mm})$ were obtained in the latero-lateral direction. The sections were stained with hematoxylin and eosin (H\&E) for histological and histometric analyses. An experienced treatment-blinded examiner (ML) selected four sections for 
immunohistochemistry using an indirect immunoperoxidase technique and the following primary antibodies: anti-tartrateresistant acid phosphatase (anti-TRAP; Santa Cruz Biotechnology); anti-proliferating cell nuclear antigen (antiPCNA; Vector Laboratories, Burlingame, CA, USA); antiosteoprotegerin (anti-OPG; Santa Cruz Biotechnology); and anti-receptor activator of nuclear factor kappa-B ligand (antiRANKL; Santa Cruz Biotechnology). Immunohistochemical procedures followed the protocol described by Gualberto et al. [31].

\section{Histological and histometric analyses}

H\&E-stained sections were analyzed under a bright field illumination optical microscope (Axiostar plus, Carl Zeiss MicroImaging GmbH 07740 Jena, Germany) to establish ABL area and characteristics of periodontal ligament and cement in the furcation region of the mandibular left first molars. The first and last sections, where the furcation region was evident, were excluded, and five equidistant sections from each specimen were selected [32]. One blinded trained examiner (ML) performed the selection. Histological analysis was conducted by a blinded and certified histologist (EE) who evaluated the magnitude of the local inflammatory response and the course of the repair process within the periodontal tissue. Another blinded examiner conducted the histometric analysis (MLFA). The area of ABL $\left(\mathrm{mm}^{2}\right)$ in the furcation region was determined using an image analysis system (AxioVision 4.8.2, Carl Zeiss MicroImaging GmbH 07740 Jena, Germany). The same examiner measured the level of $\mathrm{ABL}$ in the furcation region of each specimen section on different days to reduce variations in the data [9]. The three measurements were subjected to an analysis of compliance with a 5\% level of significance (Kappa test, 90\%). The ABL is expressed as square millimeters and represents the mean of five measurements.

\section{Immunohistochemical analysis}

Positive immunolabeling was defined as a brownish color in the cytoplasm of the cells. Quantitative analyses for TRAPand PCNA-positive cells were performed in an area of $1000 \mu \mathrm{m} \times 1000 \mu \mathrm{m}$ in the central area of the inter-radicular septum under $\times 200$ magnification. The coronary limit was the alveolar bone crest, from which it extended apically for a distance of $1000 \mu \mathrm{m}$. RANKL and OPG were analyzed throughout the furcation region in an area of $3200 \times 300 \mu \mathrm{m}$ under $\times 400$ magnification. Semi-quantitative analyses were performed in the bone and periodontal ligament of the first molar. Three histological sections from each animal were used, and the immunolabeling criteria followed Garcia et al. [10]: score 0 - no marking $(0 \%)$, score 1 -weak marking
( $<25 \%$ of cells), score 2 -moderate marking ( $<50 \%$ of cells), and score 3 - strong intensity ( $<75 \%$ of cells).

\section{Statistical analysis}

Calculation of sample size $n$ (minimum $=8$ ) demonstrated a $90 \%$ study power $(p<0.05)$. Normality and homoscedasticity of histometric and immunohistochemical data (TRAP and PCNA) were analyzed using the Shapiro-Wilk test. Histometric data and number of TRAP-positive cells were subjected to analysis of variance (ANOVA), and the significance of the results was verified using Tukey's test. Analysis of PCNA data was performed using the non-parametric method of Kruskal-Wallis followed by Student-Newman-Keuls test. All analyses were performed using software (BioEstat 5.0, Sonopress industry Brazilian, Manaus, AM, Brazil) with a $5 \%$ level of significance.

\section{Results}

\section{Histological analysis}

Connective tissue in the NT group at 7 days (Fig. 1a) was composed of a delicate network of collagen fibers, a moderate
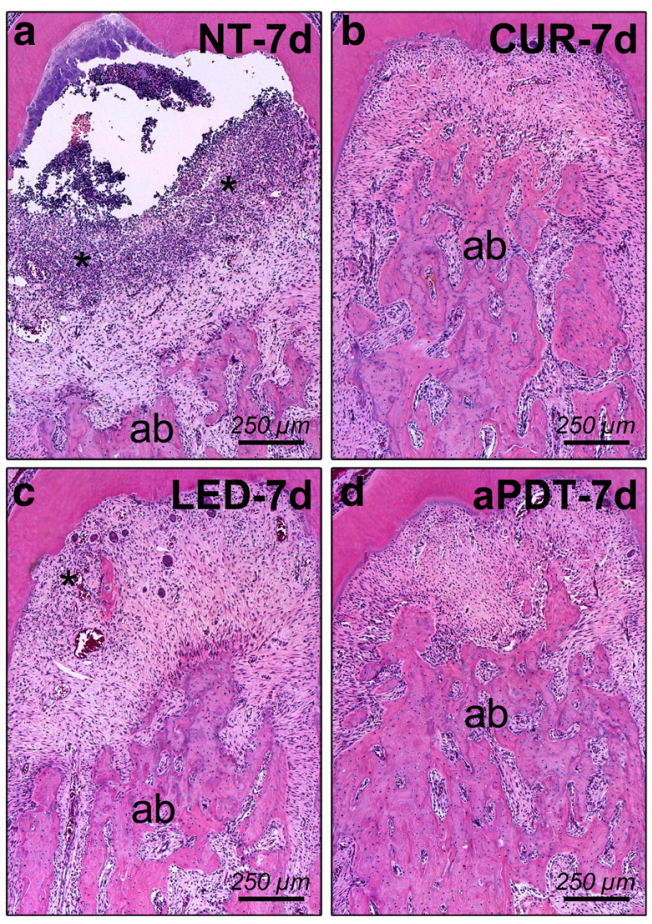

Fig. 1 Photomicrographs of the left mandibular first molar with induced periodontitis showing the degree of local inflammation, the extent of alveolar bone loss, and the periodontal repair process at 7 days in the NT (a), CUR (b), LED (c), e aPDT (d) groups. Asterisk is the inflammatory infiltrate; $a b$ alveolar bone. Original magnification: a-d: x100. Scale bars: a-d: $250 \mu \mathrm{m}$. Staining: hematoxylin and eosin (HE) 
amount of fibroblasts, and an intense inflammatory infiltrate that was composed primarily of neutrophils, macrophage, lymphocytes, and plasma cell. The inter-radicular septum was composed of thin bone trabeculae, with a highly irregular external outline in the untreated group. Histological characteristics at 15 days (Fig. 2a) were similar to the previous period, except for a reduction in the volume occupied by the inflammatory infiltrate. The connective exhibited a higher amount of collagen fibers at 30 days (Fig. 3a), but few fibroblasts and features indicated the presence of periodontitis.

An intense inflammatory infiltrate with a moderate number of fibroblasts in a thin network of collagen fibers was observed in the CUR group on day 7 (Fig. 1b). The interradicular septum was composed of fine bone trabeculae. The inflammatory infiltrate was greatly reduced with the increase in the number of fibroblasts at 15 and 30 days (Figs. 2b, 3b). A frequent thickening of bone trabeculae was also found in later periods.

The LED group exhibited connective tissue composed of a thin network of collagen fibers, a moderate amount of fibroblasts and an intense inflammatory infiltrate on day 7 (Fig. 1c). The inter-radicular septum consisted of thin bone trabeculae with a highly irregular external outline. A significant reduction of the inflammatory infiltrate with a clear volume decreased with an increased number of fibroblasts on day 15
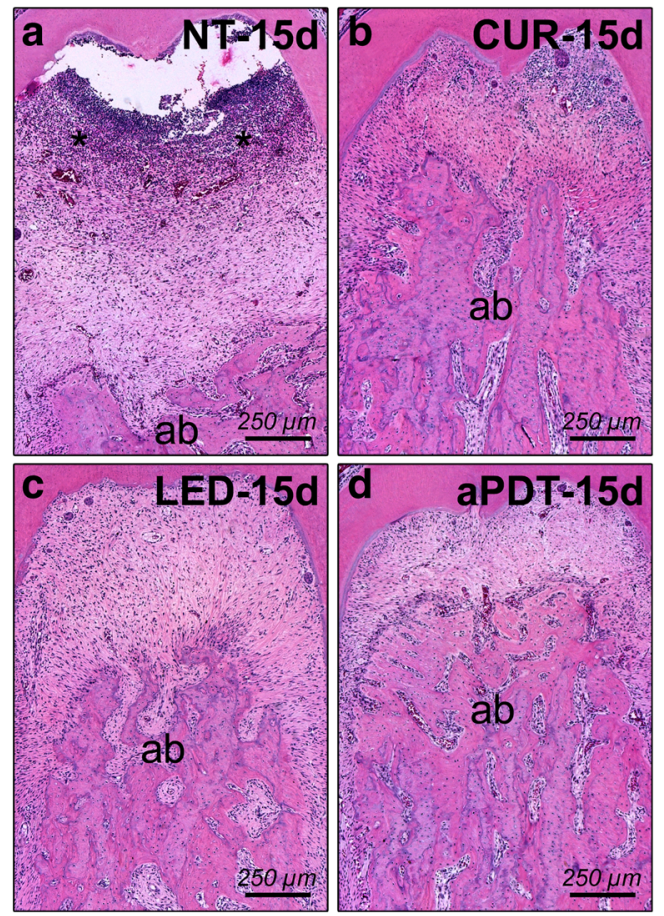

Fig. 2 Photomicrographs of the left mandibular first molar with induced periodontitis showing the degree of local inflammation, the extent of alveolar bone loss, and the periodontal repair process at 15 days in the NT (a), CUR (b), LED (c), e aPDT (d) groups. Asterisk is the inflammatory infiltrate; $a b$ alveolar bone. Original magnification: a-d: x100. Scale bars: a-d: $250 \mu \mathrm{m}$. Staining: hematoxylin and eosin (HE)
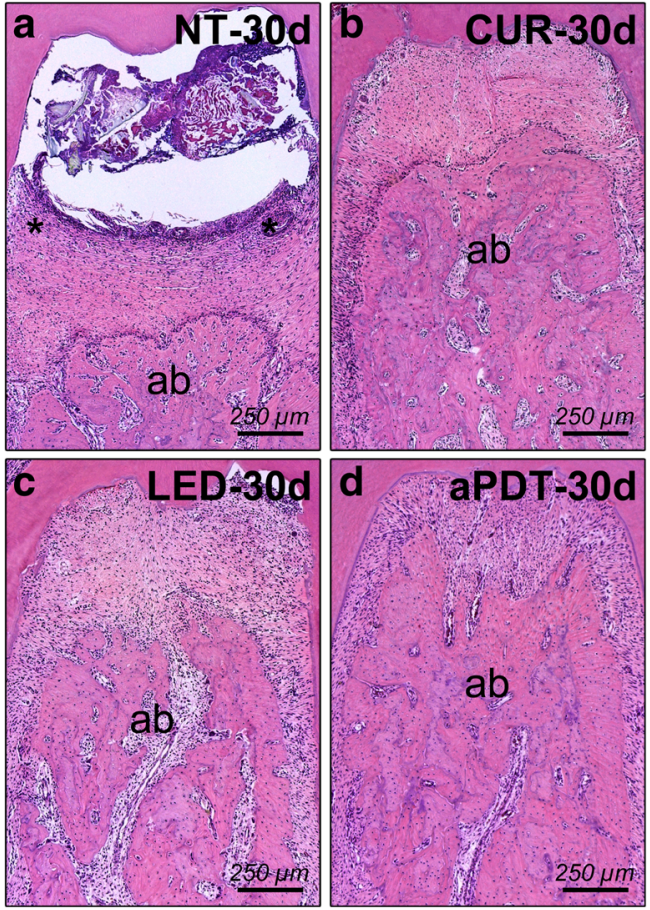

Fig. 3 Photomicrographs of the left mandibular first molar with induced periodontitis showing the degree of local inflammation, the extent of alveolar bone loss, and the periodontal repair process at 30 days in the NT (a), CUR (b), LED (c), e aPDT (d) groups. Asterisk is the inflammatory infiltrate; $a b$ alveolar bone. Original magnification: a-d: x100. Scale bars: a-d: $250 \mu \mathrm{m}$. Staining: hematoxylin and eosin (HE)

(Fig. 2c). This group showed a proportional improvement in the number of fibroblasts, a significant reduction of the inflammatory infiltrate, and a thickening of bone trabeculae at 30 days (Fig. 3c).

The aPDT group exhibited connective tissue with a moderate amount of collagen fibers that were rich in fibroblasts and a mild inflammatory infiltrate on day 7 (Fig. 1d). The volume occupied by the inflammatory infiltrate decreased significantly on day 15 (Fig. 2d). A thickening of bone trabeculae of the inter-radicular septum was also observed in the transition of these periods. The connective tissue exhibited a large amount of collagen fibers, few fibroblasts, and minimum inflammatory cells in isolated outbreaks in the furcation region at 30 days (Fig. 3d). Bone trabeculae exhibited a proportional increase in thickness.

\section{Histometric analysis}

ABL was lower in animals from the aPDT group $\left(0.8 \pm 0.62 \mathrm{~mm}^{2}\right)$ compared to the NT group $\left(1.49 \pm 0.71 \mathrm{~mm}^{2}\right)(p<0.01)$ at 7 days post-treatment. A higher ABL was also observed in animals from the NT group on days $15\left(2.05 \pm 0.17 \mathrm{~mm}^{2}\right)$ and $30\left(1.81 \pm 0.64 \mathrm{~mm}^{2}\right)$ compared to the CUR group $\left(0.55 \pm 0.21 \mathrm{~mm}^{2} ; 0.82 \pm 0.39 \mathrm{~mm}^{2}\right)$, the LED group $\left(1.14 \pm 0.39 \mathrm{~mm}^{2} ; 0.67 \pm 0.52 \mathrm{~mm}^{2}\right)$, and the aPDT 
group $\left(0.97 \mathrm{~mm}^{2} \pm 0.58,0.66 \pm 0.29 \mathrm{~mm}^{2}\right.$, respectively) during the same periods of evaluation $(p<0.01)$ (Table 1).

\section{Immunohistochemistry analysis}

Immunohistochemistry was used to detect TRAP, PCNA, OPG, and RANKL. The primary antibodies exhibited high specificity for these proteins, which was confirmed by the total absence of labeling in the negative control reactions. TRAP-positive cells were predominantly present in multinucleated osteoclasts. RANKL and OPG were expressed predominantly in osteoblasts and some fibroblasts. PCNA was located predominantly in connective tissue cells.

There was no statistically significant difference between the NT and any treatment groups for PCNA immunolabeling at 7 days $(p>0.05)$. The NT group exhibited a higher number of PCNA-positive cells at 15 days than the LED and aPDT groups. The NT group exhibited a higher number of PCNApositive cells compared to the CUR group at 30 days (Table 1; Fig. 4a, b, c, d). TRAP immunolabeling was higher in the NT group compared to the CUR and aPDT groups on day 7 . A greater number of TRAP-positive cells were found in the NT group than rats that received any treatment at 15 and 30 days $(p<0.01)$ (Table 1; Fig. 4e, f, g, h).

RANKL immunolabeling was strong (score 3 ) at 7, 15, and 30 days in the NT group and at 7 and 15 days in the CUR group. A decrease from strong to moderate in RANKL immunolabeling (score 2) was observed in the LED group at 7 and 15 days, at 30 days in the CUR group, and at 7 days in the aPDT group. The standard immunolabeling ranged from moderate (score 2) to weak (score 1) in the LED group at 30 days and at 15 and 30 days in the aPDT groups (Fig. 5a, b, c, d). A low standard (score 1) for immunolabeling of OPG prevailed in all experimental groups and periods, with the exception of specimens from the aPDT group at 30 days, which exhibited a moderate immunolabeling (score 2) (Fig. 5e, f, g, h).

\section{Discussion}

The present study evaluated the effects of aPDT as a monotherapy in periodontal treatment using curcumin as a photosensitizer and LED $(468-485 \mathrm{~nm})$ as the light source on ABL, inflammatory immune response, and bone metabolism.

Promising evidences in the literature suggest that the combination of a phenothiazine photosensitizer with a low-power laser (red) in aPDT is an adjuvant treatment of experimental periodontitis in rats [6-10, 31,32]. However, the proposal of a photosensitizer with better results remains a challenge in aPDT. The choice of curcumin as a photosensitizer is justified by its ability to be photoactivated in the presence of low wavelengths [22] and its antimicrobial action once photoactivated [25-27]. Other advantage of curcumin is its anti-inflammatory effect in periodontal tissues [33].

The results of this study revealed no benefits for the control of ABL on day 7 with LED or curcumin as monotherapy for periodontal treatment. These results suggest that ABL may be related to bacterial control failure during the initial periods in these groups. However, animals treated with aPDT had a lower ABL than animals that received no treatment. In the presence of light, curcumin can sensitize the formation of free radicals and reactive oxygen species (ROS) leading to phototoxic reactions [21]. The mechanisms leading to photokilling by curcumin have not yet been established. The photocytotoxicity of curcumin may involve one or more processes and is likely to depend strongly on where the molecule is located [21, 34]. Besides singlet oxygen formation, curcumin is a potent generator of superoxide radicals [34].

However, all treatments (CUR, LED, and aPDT) effectively controlled $\mathrm{ABL}$ in the furcation area compared to untreated animals at 15 and 30 days, but the best results were observed in the aPDT group. Similar studies demonstrated lower ABL in groups treated with aPDT using a laser $(630-680 \mathrm{~nm})$ and methylene blue or toluidine blue as the photosensitizer on
Table 1 Means and standard deviations $(\mathrm{M} \pm \mathrm{SD})$ of the histometric data for ABL in square millimeters and the number of TRAP $\left(\right.$ cell $\left./ \mathrm{mm}^{2}\right)$ and PCNA (cells $/ \mathrm{mm}^{2}$ ) in the furcation area of the left mandibular first molar in each group and time point

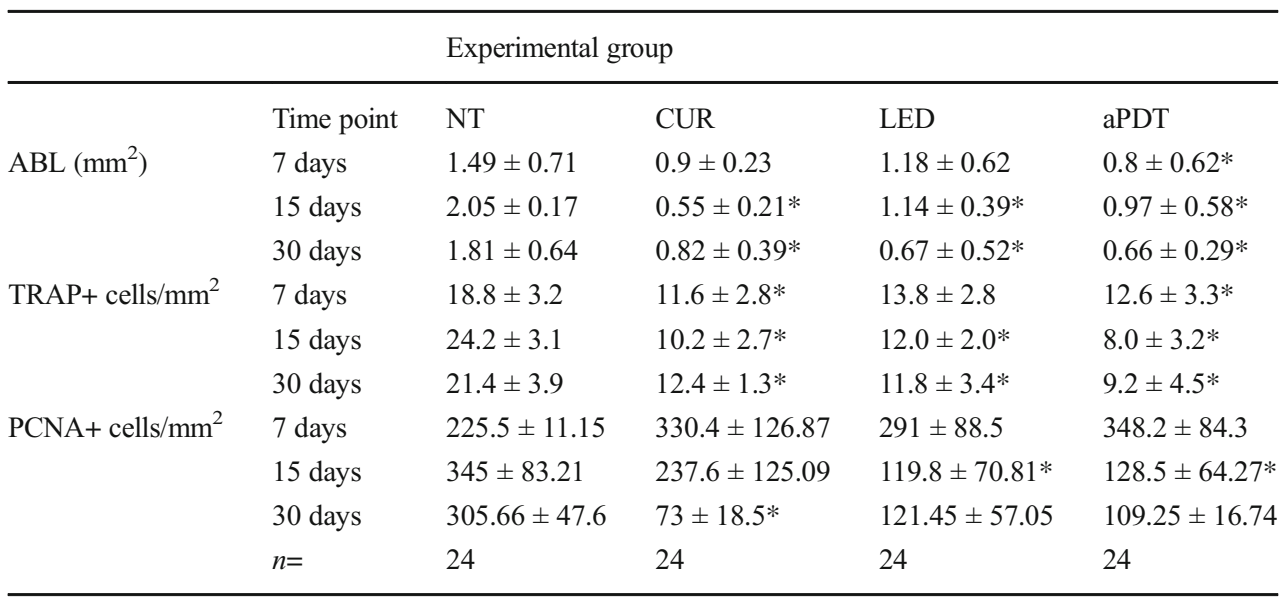

* Statistically significant difference between each treatment group compared to control group (NT) in the same time point (ANOVA and Tukey's test: $p<0.05$ ) 


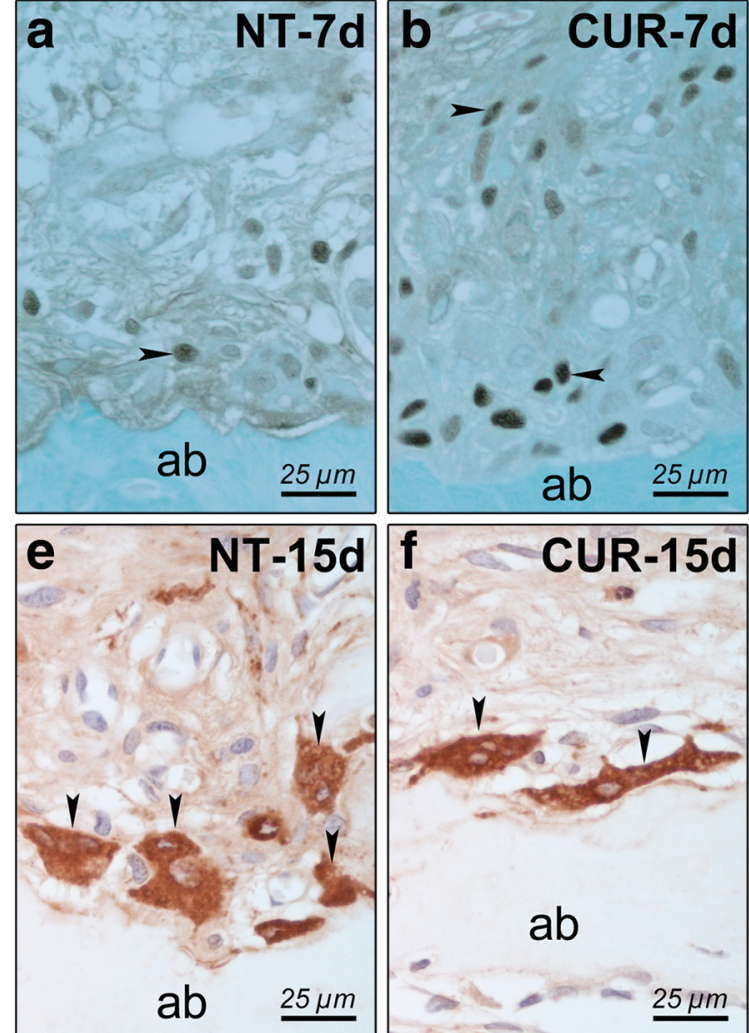

Fig. 4 Immunolabeling patterns for PCNA and TRAP. Photomicrograph showing PCNA (a-d) and TRAP (e-h) immunolabeling patterns at the furcation region of the mandibular first molar with induced periodontitis in NT (a, e), CUR $(\mathbf{b}, \mathbf{f}), \operatorname{LED}(\mathbf{c}, \mathbf{g})$, and aPDT $(\mathbf{d}, \mathbf{h})$ groups. Arrow is the

days 15 and 30, either as monotherapy [8] and adjunctive therapy [7]. The volume occupied by the inflammatory infiltrate decreased significantly in animals treated with aPDT. The inflammatory infiltrate was greatly decreased with an increase in the number of fibroblasts in animals treated with curcumin only. These results are consistent with a previous study that demonstrated the anti-inflammatory potential of curcumin in rats [33].

Decreased osteoclast activity in the furcation was observed in all treatments, which was demonstrated by a decrease in TRAP-positive cells in all periods, except animals treated with only LED on day 7. The efficacy of LEDs emitting in the $600-700 \mathrm{~nm}$ range to reduce inflammation, fibroblast proliferation, and new bone formation was demonstrated in a previous study of induced periodontitis in rats [35]. Another study in anemic animals demonstrated the potential of LEDs $(\lambda=700-20 \mathrm{~nm})$ emitting $10 \mathrm{~J} / \mathrm{cm}^{2}$ as monotherapy in repair and concluded that this irradiation caused a positive biomodulation in fibroblast proliferation [36]. The results of previous studies may explain the results of the present study on the effectiveness of treatment only in later periods ( 15 and 30 days) using $15 \mathrm{~J} / \mathrm{cm}^{2}$ as the LED, because this LED may have promoted a reduction of the inflammatory process and biomodulation of bone repair after an acute period of

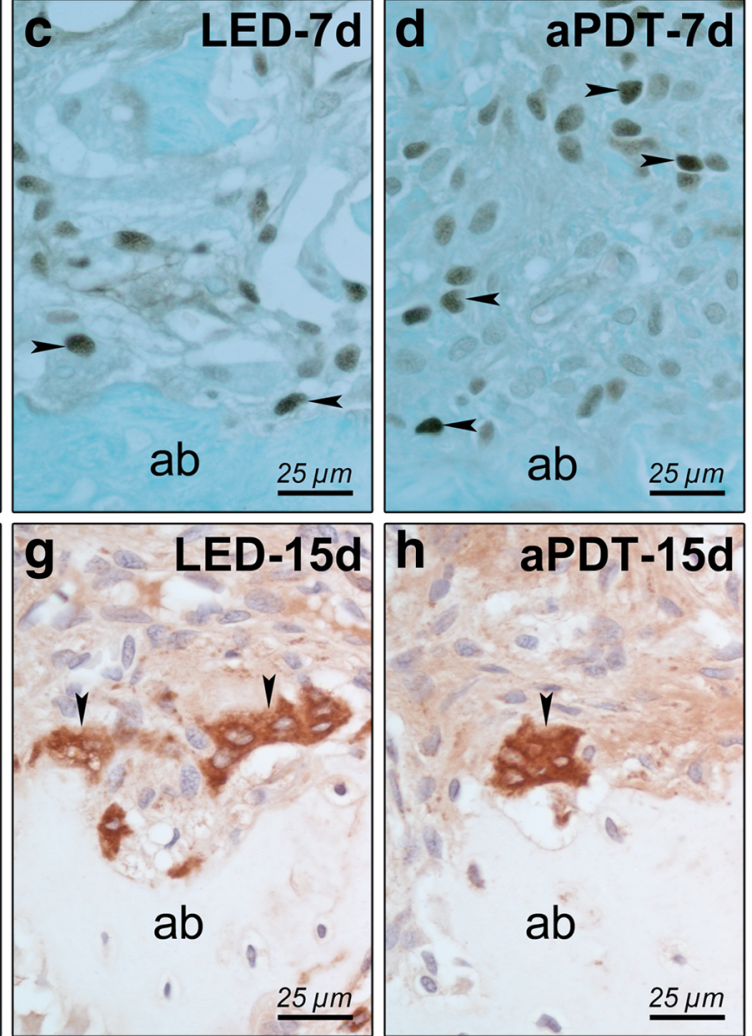

immunostained cells; $a b$ alveolar bone. Original magnification: x1000. Scale bars: a-h: $25 \mu \mathrm{m}$. Counterstaining: a-d: Fast green; e-h: Harris hematoxylin

infection. One study used the combination of eosin and LED $(440-480 \mathrm{~nm})$ with a power density of $0.69 \mathrm{~W} / \mathrm{cm}^{2}$ for $60 \mathrm{~s}$ and demonstrated a reduction in $\mathrm{ABL}$ and immunomodulation of the inflammatory response, which was observed as a reduction in neutrophil numbers. The LED used alone did not significantly reduce ABL when used as an adjuvant treatment [37].

PCNA is a cell cycle-related nuclear protein that is maximally elevated in the late G1 and S phases of proliferating cells. In the present study, there were no differences in the number of PCNA-positive cells at day 7 post-treatment. These findings are justified by the proliferative process present in all groups. However, a smaller number of PCNApositive cells were found in rats of the LED and aPDT groups when compared to rats from the NT group on day 15 . The data suggest that rats treated with either LED or aPDT were in the cell differentiation phase, while the untreated rats remained in the cell proliferation phase. These results are in agreement with the results of Ozawa et al. [38], who suggested that laser irradiation may stimulate cellular proliferation and cellular differentiation, resulting in more differentiated osteoblastic cells and increased bone formation.

A decrease in RANKL expression was observed in the treatment groups at all study time points assessed, and the 

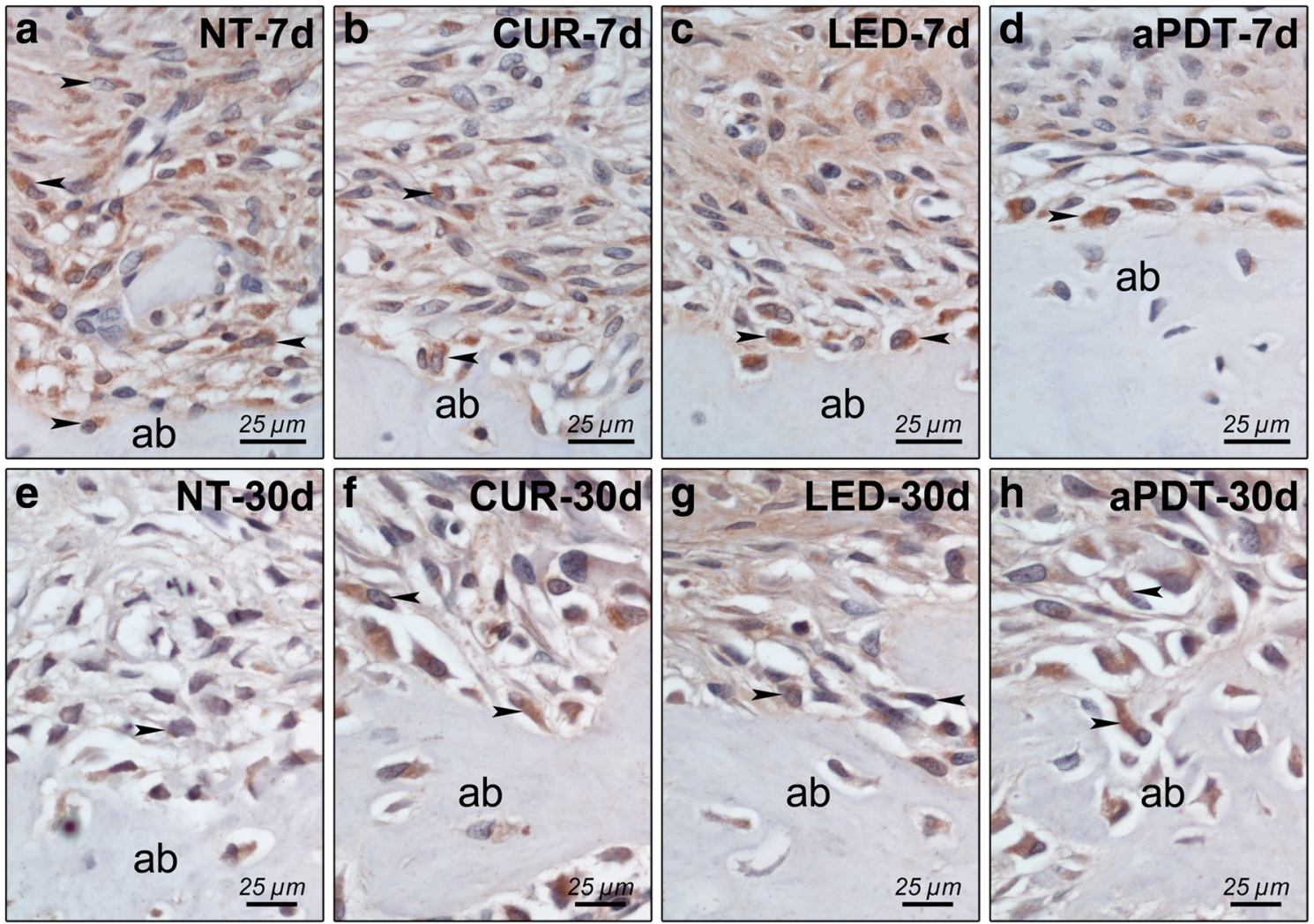

Fig. 5 Immunolabeling patterns for RANKL and OPG. Photomicrograph showing RANKL (a-d) and OPG (e-h) immunolabeling patterns at the furcation region of the mandibular first molar with induced periodontitis in NT $(\mathbf{a}, \mathbf{e}), \mathrm{CUR}(\mathbf{b}, \mathbf{f}), \mathrm{LED}(\mathbf{c}, \mathbf{g})$, and

aPDT ( $\mathbf{d}, \mathbf{h})$ groups. Arrow is the immunolabeling cells; $a b$ alveolar bone. Original magnification: $\mathrm{x} 1000$. Scale bars: $\mathrm{a}-\mathrm{h}: 25 \mu \mathrm{m}$. Counterstaining: $\mathrm{a}-\mathrm{h}$ : Harris hematoxylin

greatest effect was observed in the LED and aPDT groups. An increase in OPG-positive cells was observed on day 30 in the aPDT group, which demonstrates less osteoclastic activity in the aPDT group. These results are consistent with other studies from our research group that evaluated the effect of aPDT using phenothiazines as photosensitizers and lasers as the light source as adjuvant treatment of experimental periodontitis in rats [31, 10, 32]. Experimental studies that evaluated the effects of systemically administered curcumin on experimental periodontal disease demonstrated a low pattern of immunolabeling for RANKL and OPG when curcumin was administered intragastrically for 30 days [39]. A significant reduction in the inflammatory infiltrate and increased number of fibroblasts and collagen fibers was observed in animals that received curcumin daily via gavage for 15 days [33].

The same concentration of curcumin as the present study and an LED emitting in the range 440-460 nm effectively controlled the Candida species in vitro [27]. These authors demonstrated that 5 and $18 \mathrm{~J} / \mathrm{cm}^{2}$ effectively controlled the Candida species. The time of contact with the curcumin solution and biofilm was $1 \mathrm{~min}$ prior to irradiation in this study, which is consistent with the standard time used in other aPDT protocols $[7,9,10,31,32]$.

A recent clinical study demonstrated that the local application of a $1 \%$ curcumin gel as adjunctive therapy to periodontal treatment significantly reduced mechanically evaluated bacteria, including Pg, Prevotella intermedia, Fusobacterium nucleatum, and Capnocytophaga, compared to mechanical treatment alone [40].

Another study demonstrated that blue light irradiation alone did not alter Enterococcu faecalis viability, which is one of the bacteria responsible for the failure of endodontic treatment. Blue light-activated eosin-Y $(5 \mu \mathrm{M})$, rose bengal $(1 \mu \mathrm{M})$, or curcumin $(5 \mu \mathrm{M})$ significantly $(p<0.05)$ reduced E. faecalis viability compared to unirradiated photochemicals [41].

A previous study [42] reported a statistically significant reduction in bacterial population only when curcumin was used in combination with blue light. These authors noted that the association of curcumin followed by illumination with blue light reduced bacteria numbers in Streptococcus mutans and Lactobacillus acidophilus planktonic cultures. Reductions of 54 and $91 \%$ were obtained for 0.75 to $1.5 \mathrm{~g} / \mathrm{L}$ of curcumin, respectively. The photodynamic effect of 
curcumin was dose-dependent in dark toxicity tests, and the authors demonstrated that the tested concentrations of curcumin exhibited no toxicity in any planktonic culture. Isolated light irradiation without curcumin irrigation did not affect the viability of the microorganisms [42].

There are limitations of the animal model in representing all aspects of human periodontal disease. Nevertheless, the selection of model was necessary to optimize the goal of this study. Histologic and immunohistochemical questions cannot be typically addressed in human studies due to important ethical considerations. Rat ligature model has been used in several studies $[6,7,9,10,31,32,43]$. The ligature leads to greater accumulation of plaque and ulceration of the sulcular epithelium facilitating invasion of connective tissue [44]. As observed in human periodontitis, alveolar bone loss in the ligature model is dependent upon bacteria [43]. The destructive phase of ligature-induced experimental periodontitis is associated with a host response as shown by formation of an inflammatory infiltrate in the gingiva prior to bone resorption [44].

Evaluating the characteristics and particular effects of each proposed treatment, this study confirmed that all treatments used as a monotherapy exhibited benefits to control the inflammatory process and resorptive activity in experimental periodontitis and demonstrated the efficacy of isolated (curcumin or LED) and associated (aPDT) therapies. Though, in comparison among the groups, aPDT was more effective in controlling experimental periodontitis in rats, which can be related to the reduction of microorganisms in the biofilm using this therapy. However, the combination effects of a locally action anti-inflammatory of curcumin [33, 39 ] together with a probably vasodilator effect of LED can be as significant as the liberation of ROS or singlet oxygen on the control of experimental periodontal disease. Although the results of the present study demonstrate a significant improvement in the reduction of alveolar bone loss and a reduction of osteoclastic activity and modulation of the immuneinflammatory response in the group of the LED associated with curcumin, future studies should be performed to confirm if these findings are clinically relevant and related to the photodynamic effect.

\section{Conclusion}

Within the limits of this study, it is possible to conclude that aPDT using LED as a light source and curcumin as a photosensitizer showed effectiveness on the treatment of experimental periodontitis in rats, because it reduced inflammatory process severity, controlled bone loss by reduction of osteclastic recruitment and activity, and increased the periodontal healing.

\section{Compliance with ethical standards}

Funding The authors would like to thank the São Paulo Research Foundation ("Fundação de Amparo à Pesquisa do Estado de São Paulo", FAPESP) for the financial support to conduct this research (Process FAPESP 2012-19243-8) and the Scientific Initiation Scholarship (Process FAPESP \# 2012/ 192415 and 2012/178131). The authors also would like to thank the Scientific Initiation Scholarship Program of CNPq/PIBIC/UNESP and PROINTER-UNESP (Process \# 2016/1255).

Conflict of interest The authors declare that they have no conflict of interest.

Ethical approval All applicable international, national, and/or institutional guidelines for the care and use of animals were followed. All protocols were approved by the Animal Research Ethics Committee of the Araçatuba School of Dentistry, São Paulo State University (UNESP) in compliance with the current standards adopted by the Brazilian College of Animal Experimentation (Process \#00725-2012).

\section{References}

1. Teles R, Teles F, Frias-Lopez J, Paster B, Haffajee A (2013) Lessons learned and unlearned in periodontal microbiology. Periodontol 2000 62:95-162

2. Caton JG C, Ciancio SG, Blieden TM, Bradshaw M, Crout RJ, Hefti AF, Massaro JM, Polson AM, Thomas J, Walker C (2001) Subantimicrobial dose doxycycline as an adjunct to scaling and root planing: post-treatment effects. J Clin Periodontol 28:782-789

3. Maisch T (2007) Anti-microbial photodynamic therapy: useful in the future? Lasers Med Sci 22:83-91

4. Kömerik N, Nakanishi H, MacRobert AJ, Henderson B, Speight P, Wilson M (2003) In vivo killing of Porphyromonas gingivalis by toluidine blue-mediated photosensitization in an animal model. Antimicrob Agents Chemother 47:932-940

5. Qin YL, Luan XL, Bi LJ, Sheng YQ, Zhou CN, Zhang ZG (2008) Comparison of toluidine blue-mediated photodynamic therapy and conventional scaling treatment for periodontitis in rats. J Periodontal Res 43:162-167

6. de Almeida JM, Theodoro LH, Bosco AF, Nagata MJH, Oshiiwa M, Garcia VG (2007) Influence of photodynamic therapy on the development of ligature-induced periodontitis in rats. J Periodontol 78:566-575

7. de Almeida JM, Theodoro LH, Bosco AF, Nagata MJH, Bonfante S, Garcia VG (2008) Treatment of experimental periodontal disease by photodynamic therapy in rats with diabetes. J Periodontol 79: 2156-2165

8. de Almeida JM, Theodoro LH, Bosco AF, Nagata MJH, Oshiiwa M, Garcia VG (2008) In vivo effect of photodynamic therapy on periodontal bone loss in dental furcations. J Periodontol 79:10811088

9. Garcia VG, Longo M, Fernandes LA, Junior ECG, Dos Santos SC, Bosco AF, Nagata MJ, Theodoro LH (2013) Treatment of experimental periodontitis in rats using repeated adjunctive antimicrobial photodynamic therapy. Lasers Med Sci 28:143-150

10. Garcia VG, Longo M, Gualberto Júnior EC, Bosco AF, Nagata MJH, Ervolino E, Theodoro LH (2014) Effect of the concentration of phenothiazine photosensitizers in antimicrobial photodynamic therapy on bone loss and the immune inflammatory response of induced periodontitis in rats. J Periodontal Res 49:584-594

11. Braun A, Dehn C, Krause F, Jepsen S (2008) Short-term clinical effects of adjunctive antimicrobial photodynamic therapy in 
periodontal treatment: a randomized clinical trial. J Clin Periodontol 35:877-884

12. Polansky R, Haas M, Heschl A, Wimmer G (2009) Clinical effectiveness of photodynamic therapy in the treatment of periodontitis. J Clin Periodontol 36:575-580

13. Chondros P, Nikolidakis D, Christodoulides N, Rössler R, Gutknecht N, Sculean A (2009) Photodynamic therapy as adjunct to non-surgical periodontal treatment in patients on periodontal maintenance: a randomized controlled clinical trial. Lasers Med Sci 24:681-688

14. Pinheiro SL, Donegá JM, Seabra LM, Adabo MD, Lopes T, do Carmo TH, Ribeiro MC, Bertolini PF (2010) Capacity of photodynamic therapy for microbial reduction in periodontal pockets. Lasers Med Sci 25:87-91

15. Theodoro LH, Silva SP, Pires JR, Soares GH, Pontes AE, Zuza EP, Spolidório DM, de Toledo BE, Garcia VG (2012) Clinical and microbiological effects of photodynamic therapy associated with nonsurgical periodontal treatment. A 6-month follow-up. Lasers Med Sci 27:687-693

16. Alwaeli HA, Al-khateeb SN, Al-sadi A (2015) Long-term clinical effect of adjunctive antimicrobial photodynamic therapy in periodontal treatment: a randomized clinical trial. 30:801-807

17. Betsy J, Prasanth CS, Baiju KV, Prasanthila J, Subhash N (2014) Efficacy of antimicrobial photodynamic therapy in the management of chronic periodontitis: a randomized controlled clinical trial. J Clin Periodontol 41:573-581

18. Monzavi A, Chinipardaz Z, Mousavi M, Fekrazad R, Moslemi N, Azaripour A, Bagherpasand O, Chiniforush N (2016) Antimicrobial photodynamic therapy using diode laser activated indocyanine green as an adjunct in the treatment of chronic periodontitis: a randomized clinical trial. Photodiagn Photodyn Ther 14:93-97

19. Wainwright M (2008) Photodynamic therapy: the development of new photosensitisers. Anti Cancer Agents Med Chem 8:280-291

20. Haukvik T, Bruzell E, Kristensen S, Tonnesen HH (2009) Photokilling of bacteria by curcumin in different aqueous preparations. Studies on curcumin and curcuminoids XXXVII Pharmazie 64:666-673

21. Bruzell EM, Morisbak E, Tonnesen HH (2005) Studies on curcumin and curcuminoids. XXIX. Photoinduced cytotoxicity of curcumin in selected aqueous preparations. Photochem Photobiol Sci 4:523-530

22. Wikene KO, Hegge AB, Ellen Bruzell HHT (2015) Formulation and characterization of lyophilized curcumin solid dispersions for antimicrobial photodynamic therapy (aPDT): studies on curcumin and curcuminoids LII. Drug Dev Ind Pharm 41:969-977

23. Aggarwal BB, Deb L, Prasad S (2015) Curcumin differs from tetrahydrocurcumin for molecular targets, signaling pathways and cellular responses. Molecules 20:185-205

24. Martins CVB, da Silva DL, Neres AT, Magalhães TF, Watanabe GA, Modolo LV, Sabino AA, de Fátima A, de Resende MA (2009) Curcumin as a promising antifungal of clinical interest. J Antimicrob Chemother 63:337-339

25. Dujic J, Kippenberger S, Ramirez-Bosca A, Diaz-Alperi J, BereiterHahn J, Kaufmann R, Bernd A, Hofmann M (2009) Curcumin in combination with visible light inhibits tumor growth in a xenograft tumor model. Int J Cancer 124:1422-1428

26. Araújo NC, Fontana CR, Gerbi MEM, Bagnato VS (2012) Overallmouth disinfection by photodynamic therapy using curcumin. Photomed Laser Surg 30:96-101

27. Dovigo LN, Pavarina AC, Carmello JC, MacHado AL, Brunetti IL, Bagnato VS (2011) Susceptibility of clinical isolates of Candida to photodynamic effects of curcumin. Lasers Surg Med 43:927-934

28. Diego, Leite V, Paolillo FR, Parmesano TN, Fontana CR, Bagnato VS (2014) Effects of photodynamic therapy with blue light and curcumin as mouth rinse for oral disinfection. Photomed Laser Surg 32:627-632
29. Sreedhar A, Sarkar I, Rajan P, Pai J, Malagi S, Kamath V, Barmappa R (2015) Comparative evaluation of the efficacy of curcumin gel with and without photo activation as an adjunct to scaling and root planing in the treatment of chronic periodontitis: a split mouth clinical and microbiological study. J Nat Sci Biol Med 6:102-109

30. Paschoal MA, Moura CM, Jeremias F, Souza JF, Bagnato VS, Giusti JS, Santos-Pinto L (2015) Longitudinal effect of curcuminphotodynamic antimicrobial chemotherapy in adolescents during fixed orthodontic treatment: a single-blind randomized clinical trial study. Lasers Med Sci 30:2059-2065

31 Gualberto EC Jr, Theodoro LH, Longo M, Novaes VCN, Nagata MJH, Ervolino E, Garcia VG (2016) Antimicrobial photodynamic therapy minimizes the deleterious effect of nicotine in female rats with induced periodontitis. Lasers Med Sci 31:83-94

32 Garcia VG, Fernandes LA, Macarini VC, de Almeida JM, Martins TM, Bosco AF, Nagata MJ, Cirelli JA, Theodoro LH (2011) Treatment of experimental periodontal disease with antimicrobial photodynamic therapy in nicotine-modified rats. J Clin Periodontol 38:1106-1114

33 Guimarães MR, Coimbra LS, De Aquino SG, Spolidorio LC, Kirkwood KL, Rossa C (2011) Potent anti-inflammatory effects of systemically administered curcumin modulate periodontal disease in vivo. J Periodontal Res 46:269-279

34 Tønnesen HH, de Vries H, Karlsen J, Beijersbergen van Henegouwen G (1987) Studies on curcumin and curcuminoids. IX: Investigation of the photobiological activity of curcumin using bacterial indicator systems. J Pharm Sci 76:371-373

35 Chang PC, Chien LY, Ye Y, Kao MJ (2013) Irradiation by lightemitting diode light as an adjunct to facilitate healing of experimental periodontitis in vivo. J Periodontal Res 48:135-143

36 Oliveira Sampaio SCP, de C Monteiro JS, Cangussú MC, Pires Santos GM, dos Santos MA, dos Santos JN, Pinheiro AL (2013) Effect of laser and LED phototherapies on the healing of cutaneous wound on healthy and iron-deficient Wistar rats and their impact on fibroblastic activity during wound healing. Lasers Med Sci 28:799-806

37 Carvalho AS, Napimoga MH, Coelho-Campos J, Silva-Filho VJ, Thedei G (2011) Photodynamic therapy reduces bone resorption and decreases inflammatory response in an experimental rat periodontal disease model. Photomed Laser Surg 29:735-740

38 Ozawa Y, Shimizu N, Kariya G, Abiko Y (1998) Low-energy laser irradiation stimulates bone nodule formation at early stages of cell culture in rat calvarial cells. Bone 22:347-354

39 Zhou T, Chen D, Li Q, Sun X, Song Y, Wang C (2013) Curcumin inhibits inflammatory response and bone loss during experimental periodontitis in rats. Acta Odontol Scand 71:349-356

40 Bhatia M, Urolagin SS, Pentyala KB, Urolagin SB, Menaka KB, Bhoi S (2014) Novel therapeutic approach for the treatment of periodontitis by curcumin. J Clin Diagnostic Res 8:65-69

41 Pileggi G, Wataha JC, Girard M, Grad I, Schrenzel J, Lange N, Bouillaguet S (2013) Blue light-mediated inactivation of enterococcus faecalis in vitro. Photodiagn Photodyn Ther 10:134-140

42 Araújo NC, Fontana CR, Bagnato VS, Gerbi MEM (2012) Photodynamic effects of curcumin against cariogenic pathogens. Photomed Laser Surg 30:393-399

43 Theodoro LH, Longo M, Ervolino E, Duque C, Ferro-Alves ML, Assem NZ, Louzada LM, Garcia VG (2016) Effect of low-level laser therapy as an adjuvant in the treatment of periodontitis induced in rats subjected to 5-fluorouracil chemotherapy. J Periodontal Res 51:669-680

44 Graves DT, Fine D, Teng YT, Van Dyke TE, Hajishengallis $\mathrm{G}$ (2008) The use of rodent models to investigate hostbacteria interactions related to periodontal diseases. J Clin Periodontol 35:89-105 